\title{
Batroxobin protects against spinal cord injury in rats by promoting the expression of vascular endothelial growth factor to reduce apoptosis
}

\author{
HUI YU, BIN LIN, YONGZHI HE, WENBIN ZHANG and YANG XU \\ Department of Orthopedics, The 175th Hospital of the PLA, Southeast Hospital of Xiamen University, \\ Zhangzhou, Fujian 363000, P.R. China
}

Received July 5, 2014; Accepted March 3, 2015

DOI: $10.3892 /$ etm.2015.2368

\begin{abstract}
The host response to spinal cord injury (SCI) can lead to an ischemic environment that can induce cell death. Therapeutic interventions using neurotrophic factors have focused on the prevention of such reactions in order to reduce this cell death. Vascular endothelial growth factor (VEGF) is a potent angiogenic and vascular permeability factor. We hypothesized in this study that batroxobin would exhibit protective effects following SCI by promoting the expression of VEGF to reduce the levels of apoptosis in a rat model of SCI. Ninety adult female Sprague Dawley rats were divided randomly into sham injury (group I), SCI (group II) and batroxobin treatment (group III) groups. The Basso-Bettie-Bresnahan (BBB) scores, number of apoptotic cells and expression of VEGF were assessed at 1, 3, 5, 7, 14 and 28 days post-injury. The BBB scores were significantly improved in group III compared with those in group II between days 5 and 28 post-injury $(\mathrm{P}<0.05)$. At each time-point subsequent to the injury, the number of apoptotic cells in group III was reduced compared with that in group II. Compared with group II, treatment with batroxobin significantly increased the expression of VEGF from day 3 until 2 weeks post-SCI $(\mathrm{P}<0.05)$, while no significant difference was observed at day 28. These data suggest that batroxobin has multiple beneficial effects on SCI, indicating a potential clinical application.
\end{abstract}

\section{Introduction}

Spinal cord injury (SCI) predominantly affects younger members of the population and is caused by traffic or sports-related accidents. The condition can result in severe

Correspondence to: Dr Bin Lin, Department of Orthopedics, The 175th Hospital of the PLA, Southeast Hospital of Xiamen University, 269 Zhanghua Road, Zhangzhou, Fujian 363000, P.R. China

E-mail: linbin813@163.com

Key words: batroxobin, vascular endothelial growth factor, apoptosis, spinal cord injury neurological deficits, such as para- and quadriplegia (1). Since traumatic injury to the mammalian spinal cord exhibits a highly dynamic nature, characterized by a complex pattern of insidious, destructive biochemical and pathophysiological events, the potential for functional recovery from the condition is limited (2). Following SCI, substantial secondary damage within the tissue is caused by increased vascular permeability, infiltration of inflammatory cells and subsequent focal edema, which may induce apoptosis (3-5). Following SCI, cells at the site of injury may undergo cell death through post-traumatic necrosis or apoptosis, the latter of which can be demonstrated by nuclear DNA fragmentation and caspase activation. Apoptosis, in particular, is a prominent event in the spinal cord subsequent to SCI (6). Apoptosis has been shown to occur widely in the white matter, concurrently with Wallerian degeneration, and to affect neurons and oligodendrocytes. The apoptotic cell death of both neurons and oligodendrocytes may therefore be a causative factor contributing to the paralysis of patients with SCI $(7,8)$. Therapeutic interventions using neurotrophic factors have focused on the prevention of such reactions to reduce cell death and promote tissue regeneration (9).

Vascular endothelial growth factor (VEGF) has long been known as a potent angiogenic factor that stimulates the proliferation and migration of endothelial cells and the in vivo formation of new blood vessels (10). The association of VEGF with the central nervous system (CNS) has been predominantly studied in models of ischemic stroke or brain tumor (11); however, VEGF has also been attracting attention as a neuroprotective and neurotrophic factor involved in nerve regeneration and the promotion of functional recovery $(12,13)$. It has been found that VEGF enhances neurite outgrowth and neuroprotection, and reduces post-traumatic apoptosis following CNS injury (14-16). VEGF is crucial in a number of processes in the CNS, including vascularization, neuronal proliferation and the growth of coordinated vascular and neuronal networks (17). Accordingly, enhancing the expression of VEGF may have therapeutic potential for the treatment of SCI.

Batroxobin is a thrombin-like serine protease from the venom of the snake Bothrops moojeni that can decrease blood fibrinogen levels and promote blood flow (18). Batroxobin has been widely used clinically in various ischemic disorders, such as stroke, deep-vein thrombosis, myocardial infarction and 
peripheral arterial thrombosis (19-21); however, it has not been fully investigated whether batroxobin can exhibit protective effects by promoting the expression of VEGF to reduce apoptosis in SCI. This study was therefore designed to investigate whether batroxobin has a beneficial effect on rats with SCI and to explore the possible clinical application of batroxobin.

\section{Materials and methods}

This protocol was evaluated and approved by the Governmental Animal Care Committee of the Medical College of Xiamen University (Zhangzhou, China) and was performed according to the National Institutes of Health guidelines on the ethical use of animals. Every effort was made to minimize animal suffering and to reduce the number of animals used.

Animals and surgical procedures. Ninety adult female Sprague Dawley rats (Experimental Animal Center of Xiamen University) weighing 280-300 $\mathrm{g}$ were randomly assigned to the following three groups: Sham injury (group I, n=30), SCI (group II, n=30) and batroxobin treatment (group III, $n=30$ ). Any animals that died during the experiment were not included. Batroxobin was obtained from Nuokang Bio-Pharmaceutical, Inc. (Shenyang, China). Prior to surgery, the animals were anesthetized by intraperitoneal injection of $400 \mathrm{mg} / \mathrm{kg}$ chloral hydrate (Beyotime Institute of Biotechnology, Haimen, China). During the surgery, the rats were placed in a prone position on a warming pad to maintain a body temperature of $37.0 \pm 0.5^{\circ} \mathrm{C}$. Upon completion of the surgery, the rats were housed in individual cages with access to food and water ad libitum, and administered an intramuscular injection of 200,000 U/day penicillin (175th Hospital of the PLA, Zhangzhou, China) for 3 days.

All rats were injured at the thoracic level 12 (T12), using an established weight-drop model described in a previous study (22). Briefly, the skin and muscle overlying the spinal column were incised and a laminectomy was performed at T12, leaving the dura intact. A moderate-intensity weight-drop (10 g, $7.0 \mathrm{~cm}$ ) was performed using an impactor with a diameter of $2.5 \mathrm{~mm}$ (Xiamen University) onto the exposed T12 cord. The rats in group I were treated in an identical manner to the rats subjected to SCI with the omission of the weight-drop step.

Following the surgery, the bladders of the rats were manually pressed twice daily until spontaneous voiding occurred. The dosage of batroxobin (DF-521; Beijing Tobishi Pharmaceutical Co., Ltd., Beijing, China) was selected according to the manufacturer's instructions, which recommended 10 batroxobin units (BU) as the regular initial dose and $5 \mathrm{BU}$ as the maintenance dose. Considering the differences between humans and rats, the rats in group III were injected with batroxobin at a dosage of $5 \mathrm{BU} / \mathrm{kg} /$ day via the tail vein within $8 \mathrm{~h}$ of SCI until 3 days post-injury. Instead of batroxobin, the rats in groups I and II were administered saline through pumps as a control treatment.

Basso-Beattie-Bresnahan (BBB) evaluation of locomotion. The rats were tested for locomotor deficits at 1 day before and 1, 4 and 7 days after SCI with a standard open-field locomotor test, developed by Basso et al (23). This BBB locomotor rating scale evaluates the following criteria: Extent of joint movement, weight support and stepping/walking behavior of the hindlimbs. The rating scale ranges from 0 (no observable hindlimb movement) to 21 (normal locomotion), and scores were assigned for both hind limbs by two independent observers blinded to the experiments. The main functional outcome was calculated by the mean value.

Hematoxylin and eosin (HE) staining for the detection of pathological changes. For the histological staining, 5- $\mu \mathrm{m}$ transverse sections of injured spinal cord tissue from each group at 1,3,5, 7, 14 and 28 days post-injury were deparaffinized and placed into fresh xylene for 15 min twice. The sections were re-hydrated in $100 \%$ alcohol for 5 min twice, and then 95 and $70 \%$ alcohol once for $3 \mathrm{~min}$, respectively. The sections were subsequently washed briefly in double-distilled (dd) $\mathrm{H}_{2} \mathrm{O}$ and stained in Harris hematoxylin (Beyotime Institute of Biotechnology) solution for 5 min. Following staining, the sections were washed in running tap water for $8 \mathrm{~min}$, subjected to differentiation with $1 \%$ acid alcohol for $30 \mathrm{sec}$ and blued in $0.2 \%$ ammonia water for $30 \mathrm{sec}$. The sections were then washed in running tap water for a further $5 \mathrm{~min}$ and rinsed in $95 \%$ alcohol for $\sim 15$ dips. The sections were stained in Eosin-Phloxine solution (Beyotime Institute of Biotechnology) for 1 min, prior to undergoing 95 and $100 \%$ alcohol dehydration (5 min each) and clearing in two changes of xylene (5 min each). Finally, the sections were mounted with mounting medium (Beyotime Institute of Biotechnology). The images were captured using an FV300 confocal microscope (Olympus Corp., Tokyo, Japan).

Terminal deoxynucleotidyl-transferase-mediated dUTP nick end labeling (TUNEL) test for apoptosis. For the detection of apoptosis, TUNEL staining was performed using a TUNEL detection kit according to the manufacturer's instructions (ApopTag ${ }^{\circledR}$ horseradish peroxidase kit; DBA, Milan, Italy). Briefly, sections of SCI tissue at 1, 3, 5, 7, 14 and 28 days post-injury were immersed in xylene for $5 \mathrm{~min}$ twice at room temperature, and in 100, 90, 80 and $70 \%$ ethanol for $5 \mathrm{~min}$ twice. The sections were then incubated in $15 \mu \mathrm{g} / \mathrm{ml}$ Proteinase $\mathrm{K}$ solution for $20 \mathrm{~min}$ at room temperature and washed with phosphate-buffered saline (PBS). Hydrogen peroxide (3\%), applied for $5 \mathrm{~min}$ at room temperature, was utilized to terminate any endogenous peroxidase activity, prior to the sections being washed with PBS. The sections were then immersed in terminal deoxynucleotidyl transferase (TdT) buffer containing TdT and biotinylated dUTP, incubated in a humid atmosphere at $37^{\circ} \mathrm{C}$ for $90 \mathrm{~min}$, and washed with PBS. Subsequent to being washed, the sections were incubated at room temperature for a further $30 \mathrm{~min}$ with anti-horseradish peroxidase-conjugated antibody (GeneTex, San Antonio, Texas, USA), and 3,3'-diaminobenzidine was used to visualize the signals. The sections were then washed in $\mathrm{ddH}_{2} \mathrm{O}$ and mounted. Images were captured using an FV300 confocal microscope (Olympus Corp.).

Immunohistochemistry of VEGF. For immunohistochemical staining, each specimen was embedded in paraffin and a microtome was used to cut serial sections. VEGF immunohistochemical staining was performed using an avidin-biotin peroxidase complex technique and a Histostain ${ }^{\circledR}$ SP kit (Maixin-Bio, Inc., Fuzhou, China) in accordance with the manufacturer's instructions. Mouse monoclonal antibody 
Table I. BBB score of each group at different time-points.

\begin{tabular}{lcrr}
\hline Days post-SCI & Group I (score) & Group II (score) & Group III (score) \\
\hline 0 & $21.00 \pm 0.00$ & $21.00 \pm 0.00$ & $21.00 \pm 0.00$ \\
1 & $21.00 \pm 0.00$ & $0.69 \pm 0.24$ & $0.79 \pm 0.23$ \\
3 & $21.00 \pm 0.00$ & $2.36 \pm 0.30$ & $2.53 \pm 0.38$ \\
5 & $21.00 \pm 0.00$ & $4.82 \pm 0.31$ & $5.03 \pm 0.33^{\mathrm{a}}$ \\
7 & $21.00 \pm 0.00$ & $6.62 \pm 0.40$ & $7.55 \pm 0.37^{\mathrm{b}}$ \\
14 & $21.00 \pm 0.00$ & $7.54 \pm 0.42$ & $9.65 \pm 0.44^{\mathrm{b}}$ \\
28 & $21.00 \pm 0.00$ & $10.22 \pm 0.74$ & $13.74 \pm 0.66^{\mathrm{b}}$ \\
\hline
\end{tabular}

Compared with group II, ${ }^{\mathrm{a}} \mathrm{P}<0.01$ and ${ }^{\mathrm{b}} \mathrm{P}<0.05$. Results are presented as the mean \pm standard deviation. BBB, Basso-Beattie-Bresnahan; Group I, sham injury; Group II, SCI; Group III, SCI with batroxobin treatment; SCI, spinal cord injury.

against VEGF (1:200; \#sc-30343; Santa Cruz Biotechnology, Inc., Santa Cruz, CA, USA) and rabbit polyclonal antibody against VEGF (1:100; \#sc-33547; Santa Cruz Biotechnology, Inc.) were used for this study. Two pathologists who were unaware of the experimental data were responsible for counting the number of VEGF-positive cells in 10 high-power fields (magnification, $\mathrm{x} 400$ ) in each specimen. The average number of VEGF-positive cells per specimen was then calculated. Images were captured using an FV300 confocal microscope (Olympus Corp.).

Statistical analysis. Statistical analysis was performed using SPSS version 13.0 for Windows (SPSS, Inc., Chicago, IL, USA). Data are presented as the mean \pm standard deviation. The Mann-Whitney U-test and Spearman's rank correlation were used for the statistical analyses. $\mathrm{P}<0.05$ was considered to indicate a statistically significant difference.

\section{Results}

During surgery, the rectal temperature of the rats was maintained at $37 \pm 0.5^{\circ} \mathrm{C}$. The mean body weight of the rats in the sham surgery group was $371.8 \pm 9.7 \mathrm{~g}$ (range, 362-385 g), while the mean body weights of the control and batroxobin group rats were $375.9 \pm 7.1 \mathrm{~g}$ (range, 367-388 $\mathrm{g}$ ) and $369.0 \pm 11.3 \mathrm{~g}$ (range, 354-389 g), respectively. No significant differences in these physiological parameters existed between the groups.

Behavioral test. To evaluate the extent of motor function recovery, the BBB locomotor rating scale was used. The BBB scores were assessed for the three groups at different time-points following SCI. Table I shows the mean BBB scores of the rats in the three groups over the time-course of the experiment. Prior to surgery, the rats were all healthy (BBB score, $21 \pm 0.00$; data not shown). In the group I rats, no significant difference was observed in the hind limb movement scores measured prior to and following SCI, and the rats exhibited normal movement throughout the observation period (BBB score, 21 points). In groups II and III, the rats showed improvements in motor function at day 5 post-SCI compared with the scores on the date of the SCI; however, the average BBB score was significantly higher in the group III rats than that in the group II rats between days 5 and 28 post-SCI. On day 28, the BBB score of the group III rats was $13.74 \pm 0.66$ points, whereas the group II rats scored $10.22 \pm 0.74$ points $(\mathrm{P}<0.05)$. A BBB score of 14 is indicative of consistent weight-supported plantar steps and front-hind limb coordination (23); neither of the scores in groups II or III exceeded the 14-point threshold (mean in group III = 13.74)

\section{Histological assessments}

Visual study. Following the initial injury, tissue edema appeared immediately in the dorsal region of the spinal cord in groups II and III, while no fresh bleeding spots were observed at day 3. Scar formation was observed in the region of the lesion and conglutination with the endorhachis was apparent at days 14 and 28 post-SCI. In addition, the spinal cord was atrophic with a reduction in the diameter. In group I, areas of scar formation were observed in the region of the lesion and conglutination with the endorhachis was apparent at days 14 and 28 post-SCI. This may have been a result of the trauma of the surgery; however, no obvious edema in the spinal cord was observed and the posterior central blood vessel and the structure of the spinal cord were clearly visible.

$H \& E$ staining. At 1 day post-SCI, $\mathrm{H} \& \mathrm{E}$ staining in the group II rats showed a large area of structural damage, multifocal hemorrhage and inflammatory cell infiltration. Notably, neuron pyknosis and chromatin condensation could be observed, which indicated cell apoptosis (Fig. 1). At 14 days post-SCI, H\&E staining in the group II rats showed a small hemorrhagic focus in the gray and white matter of the spinal cord; evident destruction to the structure of the spinal cord was observed, and neurons were found to be dissolved and liquefied in the gray matter (Fig. 2). The resulting large, liquefied and necrotic area formed a cystic space. Numerous swollen axons and neovascularization were additionally observed in the white matter, and nerve fiber disorganization was apparent. Twenty-eight days after SCI, the hemorrhagic focus in the gray and white matter was almost entirely absorbed, and further destruction of the spinal cord was observed; the neurons that were dissolved and liquefied in the gray matter formed numerous vacuolar structures. Furthermore, a reduction in the inflammatory cell infiltration, and newborn disordered blood-vessels were observed (Fig. 3). Fourteen days after the SCI, H\&E staining in group III revealed damage to the structure of the spinal cord, in addition to inflammatory cell infiltration, neuron dehydration and disintegration, 


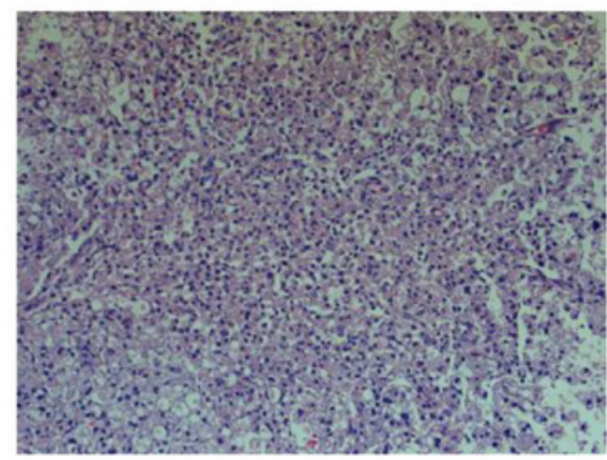

Figure 1. One day after SCI, hematoxylin and eosin staining in the SCI group showed neuron pyknosis and chromatin condensation, which indicated cell apoptosis (magnification, x100). SCI, spinal cord injury.

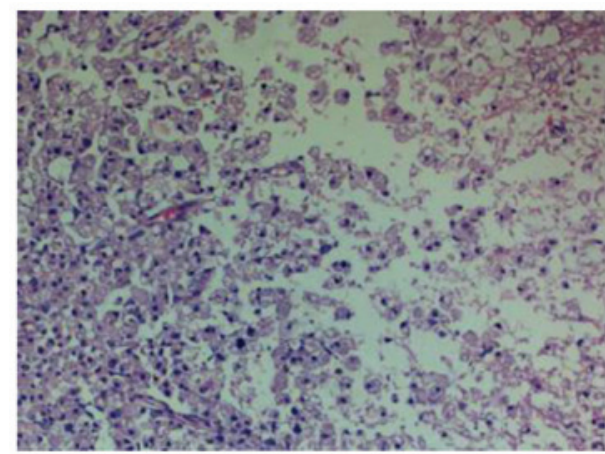

Figure 2. Fourteen days after SCI, hematoxylin and eosin staining in the SCI group showed a small hemorrhagic focus in the gray and white matter of the spinal cord and severe destruction to the structure of the spinal cord, with neurons dissolved and liquefied in the gray matter (magnification, x100). SCI, spinal cord injury.

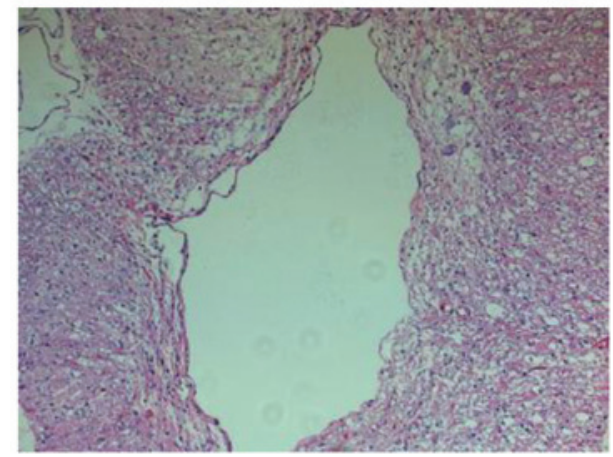

Figure 3. Twenty-eight days after SCI, hematoxylin and eosin staining in the SCI group showed that the spinal cord gray and white matter hemorrhaging had been absorbed and that the neurons had dissolved and liquefied, forming a large number of vacuolar structures. A reduction in the inflammatory infiltration, vascularization and glial scar formation were apparent, with a large number of surrounding glial cells (magnification, x100). SCI, spinal cord injury.

hyperplastic and hypertrophic gliocytes and the formation of cystic spaces; however, the damage was less severe and widespread compared with that in group II. Furthermore, fewer apoptotic cells were observed in group III than in group II. At 28 days post-SCI, it was observed that the inflammatory cell infiltration in the group III rats was reduced, and fewer

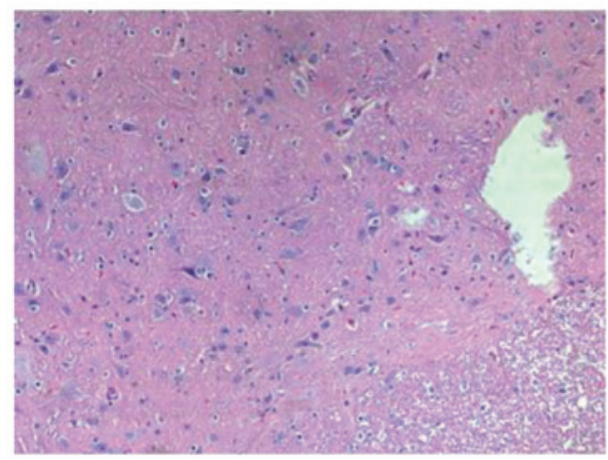

Figure 4. Twenty-eight days after SCI, hematoxylin and eosin staining in the batroxobin-treated group showed that the infiltration of inflammatory cells and amount of vascularization was reduced and that a scar had formed, although the size of the scar was smaller than that in the SCI group (magnification, x100). SCI, spinal cord injury.

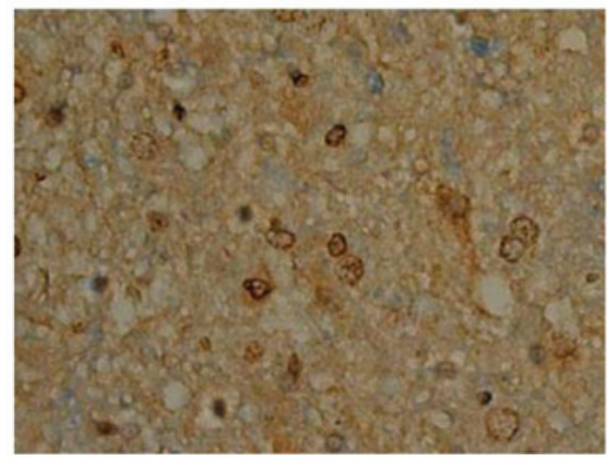

Figure 5. Terminal deoxynucleotidyl-transferase-mediated dUTP nick end labeling test for apoptosis in the sham group at 1 day after injury showed that little apoptosis had occurred (magnification, $\mathrm{x} 400$ ).

apoptotic cells were present compared with the group II rats (Fig. 4). In summary, the spinal cord pathological changes that occurred following injury were significantly attenuated by batroxobin on the 5 th, 7 th, 14 th and 28th days postoperatively.

Effect of batroxobin on cellular apoptosis in the spinal cord. SCI-induced cellular apoptosis could be detected using the TUNEL test. As shown in Fig. 5, apoptotic cells were barely detectable in group I at 1 day after SCI, as little apoptosis occurred in the absence of injury. In group II, an increased number of apoptotic cell bodies (indicated by arrows) were found at 1 day after SCI (Fig. 6), and this number continued to remain high from day 3 to day 5 (Fig. 7), prior to tapering until day 28. Compared with group II, however, a significant reduction in the number of apoptotic cell bodies (indicated by arrows) was detected in group III at 1 day after SCI (Fig. 8), and this number remained at a lower level from day 3 to day 5 (Fig. 9). Following treatment with batroxobin, the number of apoptotic cells was found to decrease significantly. This indicated that batroxobin inhibited cellular apoptosis subsequent to injury. The number of apoptotic cells in the field of view on the slides in each group was counted under a microscope and analyzed. The result of the TUNEL test indicated that the severity of tissue damage and neuronal loss was considerably milder in group III than that in group II (Table II). 


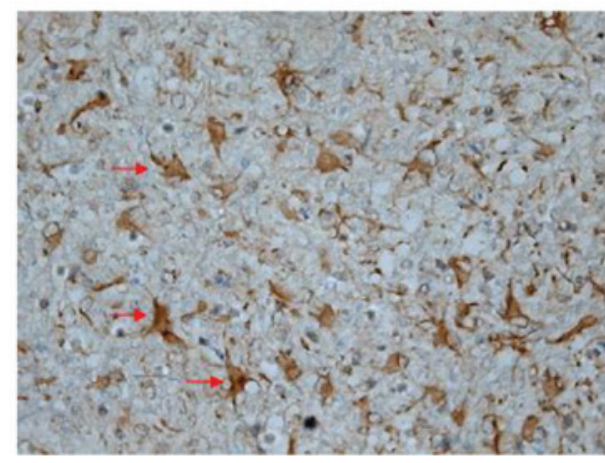

Figure 6. One day after SCI, the terminal deoxynucleotidyl-transferase-mediated dUTP nick end labeling test for apoptosis in the SCI group showed increased numbers of apoptotic cell bodies (indicated by arrows) (magnification, $\mathrm{x} 400)$. SCI, spinal cord injury.

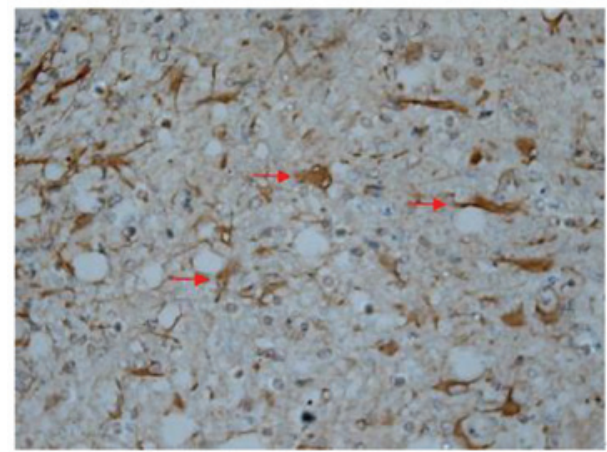

Figure 7. Five days after SCI, the terminal deoxynucleotidyl-transferase-mediated dUTP nick end labeling test for apoptosis in the SCI group showed that the number of apoptotic cell bodies (indicated by arrows) remained at a high level (magnification, x400). SCI, spinal cord injury.

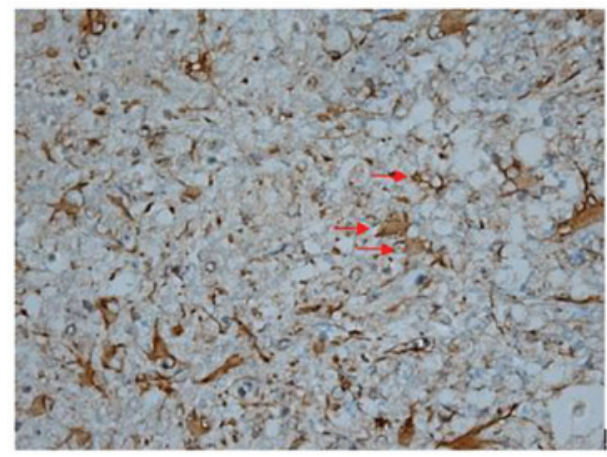

Figure 8. Terminal deoxynucleotidyl-transferase-mediated dUTP nick end labeling test for apoptosis in the batroxobin-treated group 1 day after SCI. Compared with the SCI group, a significant reduction in the number of apoptotic cell bodies (indicated by arrows) was detected (magnification, $\mathrm{x} 400$ ). SCI, spinal cord injury.

Effect of batroxobin on VEGF expression in the spinal cord. High-level constitutive expression of VEGF was observed in groups II and III; however, immunohistochemical study of VEGF in the spinal cord sections showed significant differences between the two groups. Compared with group II, batroxobin promoted the expression of VEGF between days 1 (Figs. 10 and 11) and 14 after injury. It was noted that the mean number of VEGF-positive cells per section was maximized at

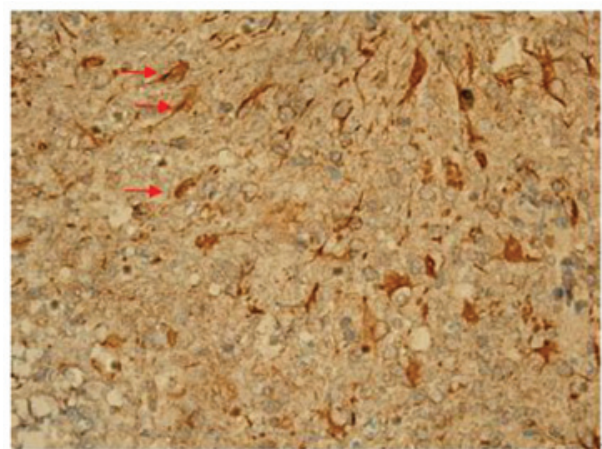

Figure 9. Terminal deoxynucleotidyl-transferase-mediated dUTP nick end labeling test for apoptosis in the batroxobin-treated group 5 days after SCI. Compared with the SCI group, the number of apoptotic cell bodies (indicated by arrows) remained at a lower level (magnification, x400). SCI, spinal cord injury.

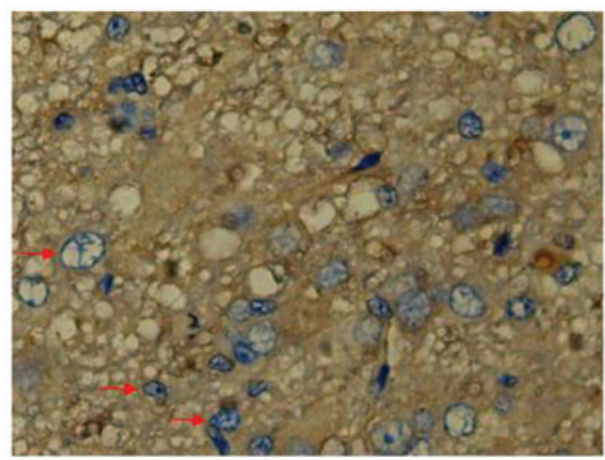

Figure 10. One day after SCI, the expression of vascular endothelial growth factor (indicated by arrows) was detected by immunohistochemistry in the SCI group (magnification, $\mathrm{x} 400$ ). SCI, spinal cord injury.

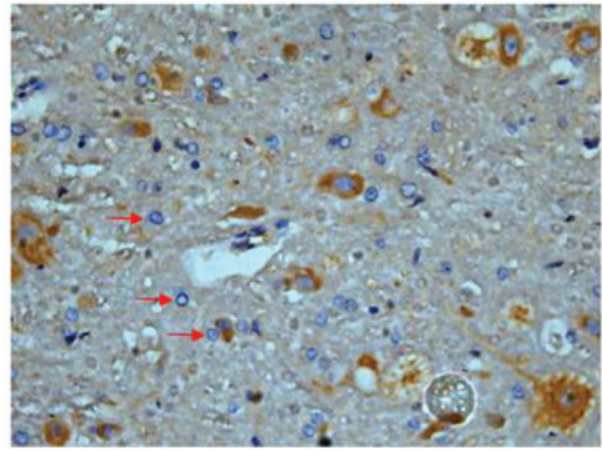

Figure 11. One day after SCI, the expression of VEGF (indicated by arrows) was detected by immunohistochemistry in the batroxobin-treated group. Compared with the SCI group, batroxobin promoted the expression of VEGF (magnification, x400). SCI, spinal cord injury; VEGF, vascular endothelial growth factor.

day 3 in groups II and III (Figs. 12 and 13). This indicated that batroxobin could promote the expression of VEGF, which played a central role in inducing angiogenesis (Table III).

Correlation of VEGF expression with the number of apoptotic cells. A significant correlation was found between the degree of VEGF expression and the number of apoptotic cells following injury $(r=-0.90052, \mathrm{P}<0.05)$. These data suggest 
Table II. TUNEL test for apoptosis detection.

\begin{tabular}{lcrr}
\hline Days post-SCI & Group I & Group II & Group III \\
\hline 1 & $15.30 \pm 0.32$ & $65.48 \pm 4.03$ & $63.65 \pm 1.58^{\mathrm{a}}$ \\
3 & $15.08 \pm 0.63$ & $49.14 \pm 2.86$ & $39.64 \pm 1.33^{\mathrm{b}}$ \\
5 & $15.03 \pm 0.40$ & $32.80 \pm 2.34$ & $28.53 \pm 1.29^{\mathrm{b}}$ \\
7 & $15.11 \pm 0.42$ & $25.04 \pm 0.83$ & $23.05 \pm 0.62^{\mathrm{b}}$ \\
14 & $15.08 \pm 0.42$ & $21.92 \pm 0.65$ & $21.53 \pm 0.47^{\mathrm{a}}$ \\
28 & $15.13 \pm 0.59$ & $21.39 \pm 0.59$ & $20.98 \pm 0.35^{\mathrm{a}}$ \\
\hline
\end{tabular}

Compared with group II, ${ }^{\mathrm{a}} \mathrm{P}<0.01$ and ${ }^{\mathrm{b}} \mathrm{P}<0.05$. Results are presented as the mean \pm standard deviation. TUNEL, terminal deoxynucleotidyl-transferase-mediated dUTP nick end labeling; Group I, sham injury; Group II, SCI; Group III, SCI with batroxobin treatment; SCI, spinal cord injury.

Table III. Number of VEGF-positive cells per section.

\begin{tabular}{llrr}
\hline Days post-SCI & Group I & Group II & Group III \\
\hline 1 & $3.66 \pm 0.03$ & $53.89 \pm 1.87$ & $55.02 \pm 1.44^{\mathrm{a}}$ \\
3 & $3.66 \pm 0.01$ & $61.35 \pm 1.89$ & $69.63 \pm 4.69^{\mathrm{b}}$ \\
5 & $3.66 \pm 0.04$ & $67.11 \pm 3.03$ & $79.10 \pm 4.61^{\mathrm{b}}$ \\
7 & $3.66 \pm 0.08$ & $59.75 \pm 1.30$ & $61.37 \pm 2.90^{\mathrm{b}}$ \\
14 & $3.66 \pm 0.01$ & $30.51 \pm 0.85$ & $40.50 \pm 1.97^{\mathrm{b}}$ \\
28 & $3.66 \pm 0.02$ & $20.08 \pm 0.35$ & $20.41 \pm 0.72$ \\
\hline
\end{tabular}

Compared with group II, ${ }^{\mathrm{a}} \mathrm{P}<0.01$ and ${ }^{\mathrm{b}} \mathrm{P}<0.05$. Results are presented as the mean \pm standard deviation. Group I, sham injury; Group II, SCI; Group III, SCI with batroxobin treatment; SCI, spinal cord injury; VEGF, vascular endothelial growth factor.

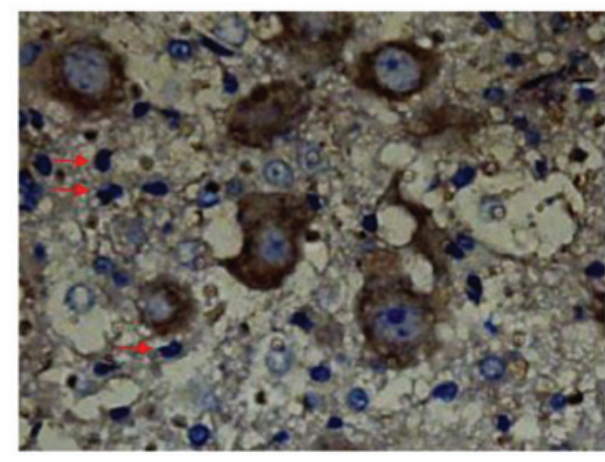

Figure 12. Three days after SCI, the expression of vascular endothelial growth factor (indicated by arrows) was maximized in the SCI group (magnification, x400). SCI, spinal cord injury.

that batroxobin may exert protective effects by promoting the expression of VEGF in order to reduce apoptosis in SCI in rats (Fig. 14).

\section{Discussion}

SCI is a serious and common CNS trauma, leading to irreversible damage to the sensory and motor functions. At present, treatment strategies for patients with SCI have been focusing increasingly on the surgical stabilization of the initial injury to prevent further loss of neurological function, without much attention being paid to nerve cell protection and a reduction of

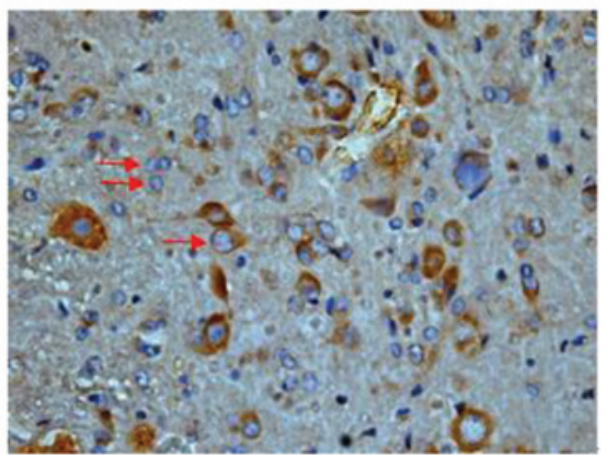

Figure 13. Three days after SCI, the expression of VEGF (indicated by arrows) was maximized in the batroxobin-treated group. Compared with the SCI group, batroxobin significantly promoted the expression of VEGF (magnification, $\mathrm{x} 400$ ). SCI, spinal cord injury; VEGF, vascular endothelial growth factor.

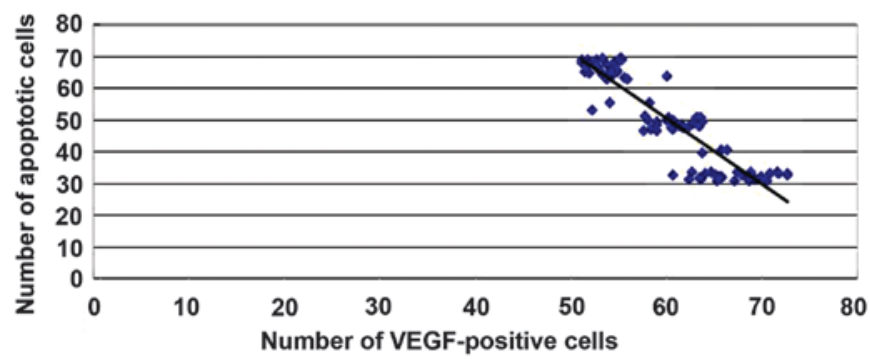

Figure 14. A significant correlation was found between the degree of VEGF expression and the number of apoptotic cells following injury ( $r=-0.90052$, $\mathrm{P}<0.05)$. VEGF, vascular endothelial growth factor. 
cell death, as has been a focus for the treatment of stroke (24). In a previous study, the potential of the nervous system to adapt to SCI from a functional (neuronal plasticity) and a structural (neuronal remodeling) perspective was demonstrated (25). However, following the primary SCI, a secondary injury expands continuously for $\sim 4$ weeks; understanding the mechanism and finding measures to control this secondary injury are of great importance. Following SCI, the response of the host can generate an ischemic environment that can lead to cell death. Furthermore, this ischemic environment limits cell transplantation approaches that could be used to promote spinal cord regeneration (26). It has been widely accepted that apoptosis is the most common form of cell death following SCI. The number of apoptotic cells is dependent on a number of factors, including external stimulation, injury severity, secondary edema and ischemia (27). By focusing on the regulation of apoptosis subsequent to SCI it has been found that the inhibition of this apoptosis could effectively protect the nerve cells (28).

A previous study has shown that enhancing spinal cord blood circulation reduces the secondary injury (29). The delivery of angiogenic factors, such as VEGF, from poly(lactide-co-glycolide) scaffolds formed by the gas foaming process can induce a local increase in blood vessel formation $(30,31)$. VEGF signals are considered to act as neurotrophic factors $(10,13,32)$. Previous studies have demonstrated the direct neurotrophic effects of VEGF on peripheral nerves (33) and reported increased neuron density and viability in mesencephalic explant cultures treated with VEGF (34). Successful neurotrophic or neuroprotective and tissue-sparing effects have also been observed following VEGF treatment in traumatic SCI $(35,36)$. Tissue edema is one of the main causes of secondary damage subsequent to SCI $(37,38)$. The application of VEGF following SCI can decrease vascular permeability and tissue edema in the spinal cord, and alleviate the deterioration of functional recovery $(39,40)$.

The aim of the present study was to examine the effect of batroxobin, a drug widely used in various ischemic disorders $(19,20)$, in reducing the secondary damage following SCI. One of the concerns for batroxobin administration in SCI is the possibility of inducing bleeding in the injured cord. It has been reported that a downstream product of batroxobin, fibrinopeptide-A, forms an unstable clot and even shortens the bleeding time in vivo $(18,41)$. In the present study, batroxobin effectively increased the expression of VEGF and reduced the number of apoptotic cells, which suggests that the batroxobin has a positive effect on the secondary damage following SCI, promoting neuronal survival and improving locomotor recovery.

In conclusion, this study has underlined the potential of batroxobin for improving the functional outcome following SCI. Since batroxobin is clinically widely used, its beneficial effect in reducing SCI can be utilized in therapeutic strategies; however, future studies are required to detail the mechanisms underlying the batroxobin-induced decrease in apoptosis and the promotion of functional recovery following SCI.

\section{References}

1. Cao HQ and Dong ED: An update on spinal cord injury research. Neurosci Bull 29: 94-102, 2013.
2. McEwen ML, Sullivan PG, Rabchevsky AG and Springer JE: Targeting mitochondrial function for the treatment of acute spinal cord injury. Neurotherapeutics 8: 168-179, 2011.

3. Inman DM and Steward O: Physical size does not determine the unique histopathological response seen in the injured mouse spinal cord. J Neurotrauma 20: 33-42, 2003.

4. Mautes AE, Weinzierl MR, Donovan F and Noble LJ: Vascular events after spinal cord injury: Contribution to secondary pathogenesis. Phys Ther 80: 673-687, 2000.

5. Kwon BK, Tetzlaff W, Grauer JN, Beiner J and Vaccaro AR: Pathophysiology and pharmacologic treatment of acute spinal cord injury. Spine J 4: 451-464, 2004.

6. Byrnes KR, Stoica BA, Fricke S, Di Giovanni S and Faden AI: Cell cycle activation contributes to post-mitotic cell death and secondary damage after spinal cord injury. Brain 130: 2977-2992, 2007.

7. Mattson MP: Apoptosis in neurodegenerative disorders. Nat Rev Mol Cell Biol 1: 120-129, 2000.

8. Mizuno Y, Mochizuki H, Sugita Y and Goto K: Apoptosis in neurodegenerative disorders. Intern Med 37: 192-193, 1998.

9. Lewis KM, Turner RJ and Vink R: Blocking neurogenic inflammation for the treatment of acute disorders of the central nervous system. Int J Inflamm 2013: 578480, 2013.

10. Sondell M, Lundborg G and Kanje M: Vascular endothelial growth factor has neurotrophic activity and stimulates axonal outgrowth, enhancing cell survival and Schwann cell proliferation in the peripheral nervous system. J Neurosci 19: 5731-5740, 1999.

11. Schoch HJ, Fischer S and Marti HH: Hypoxia-induced vascular endothelial growth factor expression causes vascular leakage in the brain. Brain 125: 2549-2557, 2002.

12. Pereira Lopes FR, Lisboa BC, Frattini F, et al: Enhancement of sciatic nerve regeneration after vascular endothelial growth factor (VEGF) gene therapy. Neuropathol Appl Neurobiol 37: 600-612, 2011

13. Jin K, Zhu Y, Sun Y, Mao XO, Xie L and Greenberg DA: Vascular endothelial growth factor (VEGF) stimulates neurogenesis in vitro and in vivo. Proc Natl Acad Sci USA 99: 11946-11950, 2002.

14. Herrera JJ, Nesic O and Narayana PA: Reduced vascular endothelial growth factor expression in contusive spinal cord injury. J Neurotrauma 26: 995-1003, 2009.

15. Sakowski SA, Heavener SB, Lunn JS, Fung K, Oh SS, Spratt SK, Hogikyan ND and Feldman EL: Neuroprotection using gene therapy to induce vascular endothelial growth factor-A expression. Gene Ther 16: 1292-1299, 2009.

16. Ma Y, Liu W, Wang Y, Chao X, Qu Y, Wang K and Fei Z: VEGF protects rat cortical neurons from mechanical trauma injury induced apoptosis via the MEK/ERK pathway. Brain Res Bull 86: 441-446, 2011.

17. Storkebaum E, Lambrechts D and Carmeliet P: VEGF: Once regarded as a specific angiogenic factor, now implicated in neuroprotection. BioEssays 26: 943-954, 2004.

18. You WK, Choi WS, Koh YS, Shin HC, Jang Y and Chung KH: Functional characterization of recombinant batroxobin, a snake venom thrombin-like enzyme, expressed from Pichia pastoris. FEBS Lett 571: 67-73, 2004.

19. Bell WR Jr: Defibrinogenating enzymes. Drugs 54 (Suppl 3): $18-31,1997$

20. Gusev EI, Skvortsova VI, Suslina ZA, Avakian GN, Martynov MIu, Temirbaeva SL, Tanashian MA, Kamchtnov PR, Stakhovskaia LV and Efremova NM: Batroxobin in patients with ischemic stroke in the carotid system (the multicenter study). Zh Nevrol Psikhiatr Im S S Korsakova 106: 31-34, 2006 (In Russian).

21. Shiraishi T, Kubo T and Matsunaga T: Chronological study of recovery of sudden deafness treated with defibrinogenation and steroid therapies. Acta Otolaryngol 111: 867-871, 1991.

22. Black P, Markowitz RS, Damjanov I, Finkelstein SD, Kushner H, Gillespie J and Feldman M: Models of spinal cord injury: Part 3. Dynamic load technique. Neurosurgery 22: 51-60, 1988.

23. Basso DM, Beattie MS and Bresnahan JC: Graded histological and locomotor outcomes after spinal cord contusion using the NYU weight-drop device versus transection. Exp Neurol 139: 244-256, 1996.

24. Goldsmith HS: The evolution of omentum transposition: From lymphedema to spinal cord, stroke and Alzheimer's disease. Neurol Res 26: 586-593, 2004.

25. Yiu G and He Z: Glial inhibition of CNS axon regeneration. Nat Rev Neurosci 7: 617-627, 2006

26. Wang Y, Gu J, Feng X, Wang H, Tao Y and Wang J: Effects of Nogo-A receptor antagonist on the regulation of the Wnt signaling pathway and neural cell proliferation in newborn rats with hypoxic ischemic encephalopathy. Mol Med Rep 8: 883-886, 2013. 
27. Beattie MS, Hermann GE, Rogers RC and Bresnahan JC: Cell death in models of spinal cord injury. Prog Brain Res 137: 37-47, 2002.

28. Wang Y, Gu J, Wang J, et al: BDNF and NT-3 expression by using glucocorticoid-induced bicistronic expression vector pGC-BDNF-IRES-NT3 protects apoptotic cells in a cellular injury model. Brain Res 1448: 137-143, 2012.

29. Jia LY, Yao AH, Kuang F, Zhang YK, Shen XF and Ju G: Beneficial effect of the traditional chinese drug shu-xue-tong on recovery of spinal cord injury in the rat. Evid Based Complement Alternat Med 2011: 862197, 2011.

30. Ennett AB, Kaigler D and Mooney DJ: Temporally regulated delivery of VEGF in vitro and in vivo. J Biomed Mater Res A 79: 176-184, 2006.

31. Peters MC, Polverini PJ and Mooney DJ: Engineering vascular networks in porous polymer matrices. J Biomed Mater Res 60: 668-678, 2002

32. Sun Y, Jin K, Xie L, Childs J, Mao XO, Logvinova A and Greenberg DA: VEGF-induced neuroprotection, neurogenesis, and angiogenesis after focal cerebral ischemia. J Clin Invest 111: 1843-1851, 2003.

33. Sondell M, Sundler F and Kanje M: Vascular endothelial growth factor is a neurotrophic factor which stimulates axonal outgrowth through the flk-1 receptor. Eur J Neurosci 12: 4243-4254, 2000.

34. Silverman WF, Krum JM, Mani N and Rosenstein JM: Vascular, glial and neuronal effects of vascular endothelial growth factor in mesencephalic explant cultures. Neuroscience 90: 1529-1541, 1999.
35. Facchiano F, Fernandez E, Mancarella S, Maira G, Miscusi M, D'Arcangelo D, Cimino-Reale G, Falchetti ML, Capogrossi MC and Pallini R: Promotion of regeneration of corticospinal tract axons in rats with recombinant vascular endothelial growth factor alone and combined with adenovirus coding for this factor. J Neurosurg 97: 161-168, 2002.

36. Widenfalk J, Lipson A, Jubran M, Hofstetter C, Ebendal T, Cao Y and Olson L: Vascular endothelial growth factor improves functional outcome and decreases secondary degeneration in experimental spinal cord contusion injury. Neuroscience 120: 951-960, 2003.

37. Narayana PA, Grill RJ, Chacko T and Vang R: Endogenous recovery of injured spinal cord: Longitudinal in vivo magnetic resonance imaging. J Neurosci Res 78: 749-759, 2004.

38. Maikos JT and Shreiber DI: Immediate damage to the blood-spinal cord barrier due to mechanical trauma. J Neurotrauma 24: 492-507, 2007.

39. Patel CB, Cohen DM, Ahobila-Vajjula P, Sundberg LM, Chacko T and Narayana PA: Effect of VEGF treatment on the blood-spinal cord barrier permeability in experimental spinal cord injury: Dynamic contrast-enhanced magnetic resonance imaging. J Neurotrauma 26: 1005-1016, 2009.

40. Sundberg LM, Herrera JJ and Narayana PA: In vivo longitudinal MRI and behavioral studies in experimental spinal cord injury. J Neurotrauma 27: 1753-1767, 2010.

41. Adams RA, Passino M, Sachs BD, Nuriel T and Akassoglou K: Fibrin mechanisms and functions in nervous system pathology. Mol Interv 4: 163-176, 2004. 